\title{
Specificity and transfer effects in time production skill: examining the role of attention
}

\author{
Erica L. Wohldmann • Alice F. Healy • \\ Lyle E. Bourne Jr.
}

Published online: 1 February 2012

(C) Psychonomic Society, Inc. 2012

\begin{abstract}
Two experiments examined transfer of a prospective, time production skill under conditions involving changes in concurrent task requirements. Positive transfer of the time production skill might be expected only when the attentional demands of the concurrent task were held constant from training to test. However, some positive transfer was found even when the concurrent task at retraining was made either easier or more difficult than the concurrent task learned during training. The amount and direction of transfer depended more on the pacing of the stimuli in the secondary task than on the difficulty of the secondary task, even though difficulty affects attentional demands more. These findings are consistent with the procedural reinstatement principle of skill learning, by which transfer from one task to another depends on an overlap in procedures required by the two skills.
\end{abstract}

Keywords Dual-task performance $\cdot$ Temporal processing

Because they are highly adaptable, human beings can usually find ways to cope with the ever-changing environmental demands that they encounter. Previous research, however, has often demonstrated strong specificity of learning to the information, skills, and context presented during acquisition. That is, even when retention of knowledge and skills is high when there is no change in the requirements

E. L. Wohldmann $(\bowtie)$

Psychology Department, California State University,

18111 Nordhoff St.,

Northrdige, CA 91330-8255, USA

e-mail: erica.wohldmann@csun.edu

A. F. Healy $•$ L. E. Bourne Jr.

University of Colorado,

Boulder, CO, USA between the learning and testing phases, transfer is low when there is a change in the task requirements between the learning and testing phases. A number of theoretical proposals have been put forward to explain specificity of learning, or weak transfer (e.g., Morris, Bransford, \& Franks, 1977; Roediger, Weldon, \& Challis, 1989; Tulving \& Thomson, 1973). Healy and Bourne (1995) explained their findings of specificity by proposing the procedural reinstatement principle, according to which transfer of a learned skill is best when the procedures used are the same during training and testing.

Healy, Wohldmann, Parker, and Bourne (2005) examined the procedural reinstatement principle in a dual-task situation in which participants were trained and later retrained on a prospective time production task, in which they generated time intervals in fixed arbitrary units. This laboratory task resembles many everyday situations in which individuals have to monitor the passage of time without consulting a clock, such as when a speaker needs to adjust the duration of his or her presentation to the given time constraints. During training, while producing time intervals, participants performed a difficult secondary task (alphabet production) or no secondary task (control). Perfect retention of the time production skill was found across a 1-week delay when the secondary-task requirements did not change between training and retraining. Strong specificity of learning was evident, however, when the secondary-task requirements were changed, even when the secondary task, if present during training, was absent during retraining. Thus, despite the additional requirements of a concurrent task, all participants showed significant improvement on the time production task during training, but learning of that skill was highly specific to the training conditions.

Healy et al. (2005) proposed that the specificity (i.e., lack of transfer) found in their study could be attributed to the 
development of a strategy that involved task integration, which they referred to as the functional task principle. More specifically, with repeated practice, participants learned to integrate procedures required to perform each of the two tasks (time production and alphabet production) simultaneously. Despite the nonrythmic nature of the alphabet production task, participants used that task as a basis for estimating the passage of time-for example, by stopping after a specific number of letters had been counted or after a given letter had been reached. Therefore, after training, the primary and secondary tasks were not treated by participants as separate and independent but were, instead, treated as a fully integrated set of requirements, or a single functional task (see also Hsiao \& Reber, 2001; Rah, Reber, \& Hsiao, 2000; Schmidtke \& Heuer, 1997; but see Ruthruff, Van Selst, Johnston, \& Remington, 2006). When conditions during retraining did not match those during training, regardless of whether the alphabet task was added or eliminated during retraining, the strategy used to perform the acquired functional task no longer applied. This change resulted in performance that was no better at the start of retraining than at the start of training. The study by Healy et al. provided only limited evidence concerning the conditions under which task integration occurs and its relationship to the occurrence of specificity or transfer of learning. A more recent study (Wohldmann, Healy, \& Bourne, 2010) manipulated separately the difficulty of the primary and secondary tasks and yielded results consistent with predictions derived from the functional task principle. That is, participants appeared to use the alphabet production task as a means of gauging the passage of time even though the rate at which they produced letters increased across trials.

It might not be surprising that changes in task requirements between training and retraining affected performance because, as with many skills, making temporal judgments involves cognitive processes that are context dependent and highly sensitive to the conditions under which they are made (e.g., Allan, 1979; Block, 1985; Clausen, 1950; Hicks, Miller, \& Kinsbourne, 1976; Wallace \& Rabin, 1960; Zakay \& Fallach, 1984). In fact, according to attentional models of time production, such as the attentional gate model (e.g., Zakay \& Block, 1997) and the attentional allocation model (e.g., Brown, 1997), requirements of a secondary task deplete the resources available to perform a concurrent time production task, although the mechanisms of depletion differ between the models. Thus, according to these models, the lack of transfer found by Healy et al. (2005) could be expected because the cognitive demands, or attentional requirements, of the secondary task were changed between training and retraining. However, by these accounts, transfer would be expected when any alterations of the concurrent task do not involve changing the attentional resources needed to perform both tasks simultaneously, as in the case, for example, when only the modality (e.g., auditory, visual) of the secondary-task stimuli changes.

The purpose of the present study was to examine further how changes in a secondary task influence primary-task performance under conditions that promote task integration and to explore the relationship between task integration and specificity or transfer of learning. As was suggested earlier, research on time perception has shown that temporal experiences are strongly influenced by attentional demands (e.g., Brown \& Boltz, 2002). For example, imposing task requirements that divide attentional resources between time production and a concurrent nontemporal task influences response bias (i.e., either over- or underestimation; see, e.g., Hicks et al., 1976, for a review of information processing during time estimation of empty and filled intervals). A lack of transfer on the time production skill across concurrent secondary tasks could be explained easily if changes in those concurrent tasks also result in changes in the attentional resources available for time production (see, e.g., Posner \& Boies, 1971). That is, if different amounts of attentional resources are available for time production during training and retraining because of differences in the difficulty of the secondary tasks, a high degree of transfer might not be expected.

If transfer of learning is primarily a matter of how attentional resources are allocated, transfer should be evident to the extent that the attentional demands of the concurrent secondary task are held constant from one secondary task to another. ${ }^{1}$ The present study directly tested this idea for the time production skill by manipulating the attentional demands of the secondary-task requirements. More specifically, two aspects of the tasks were varied, one (modality) that in itself should not affect attentional demands and another (difficulty) that should greatly affect attentional demands. The modality of the secondary task was varied by asking participants either to count tones that were played aloud or to count circles that appeared on the screen. The level of difficulty of the secondary task was varied by manipulating memory load, thus making some tasks relatively easy because of a low load (i.e., count the total number of tones or circles) and others more difficult because of a memory load twice as large (i.e., count separately both the number of high- and the number of low-pitched tones or of large and of small circles). ${ }^{2}$ In some cases, the attentional

\footnotetext{
${ }^{1}$ Note, however, that the mere switching from one secondary task to another, even when the two secondary tasks have the same attentional demands, might incur additional attentional demands (see Rubinstein, Meyer, \& Evans, 2001).

${ }^{2}$ Note that, unlike other studies, such as one by Doane, Alderton, Sohn, and Pellegrino (1996), the manipulation of difficulty here is a direct manipulation of memory load, rather than, say, a manipulation of stimulus similarity or discriminability. In the present study, participants had a memory load of either one count or two counts, with the actual stimuli and their number held constant.
} 
demands of the secondary task were different during training and retraining (when there was a change in secondary-task difficulty), but in other cases, the attentional demands were presumably the same during training and retraining (when there was a change only in secondary-task modality). Transfer of the time production skill was not expected when participants were trained with one concurrent task and retrained with another concurrent task that differed with respect to the level of attention required to perform that task.

In contrast, according to the procedural reinstatement hypothesis (Healy \& Bourne, 1995), positive transfer might be expected even when the level of attentional demands required to perform the primary and secondary tasks is changed, but only to the extent that the procedures used to perform the task combination remain constant. In Experiment 1 , the pacing of the tones and circles to be counted was kept constant across modalities and across levels of difficulty (i.e., there was a perfect mapping of small circles to highpitched tones and large circles to low-pitched tones). If the counting procedure used as a basis for time production relates to the pacing of the items shown during the secondary task, and not to either the modality or the attentional requirements of the secondary task, transfer of time production would be expected across all secondary-task conditions (i.e., across changes in levels of difficulty, as well as across changes in modality of the secondary task).

It is important to note that only the difficulty and modality of the secondary counting task were manipulated in these experiments, not the characteristics of the primary time production task. Thus, any effects of the manipulations on time production accuracy would presumably reflect a task integration strategy used by participants, in which the time production task was based, at least to some extent, on the results of the counting task.

\section{Experiment 1}

Healy et al. (2005) found essentially no transfer of a time production skill when major changes between training and retraining were made by either adding or eliminating a secondary task. To encourage transfer of the time production skill, Experiment 1 employed more modest changes between training and retraining by modifying either the modality or the difficulty of the secondary task. The pacing of the secondary tasks was held constant across changes in modality and difficulty, and this constant pacing could promote transfer from training to retraining. As has been noted, theories based on attentional demands and procedural reinstatement yield different expectations about the effect of manipulating the secondary task at training and testing.
During training, participants performed a time production task along with a concurrent secondary task that involved counting either circles or tones. ${ }^{3}$ The secondary tasks used here all involved explicit counting, ensuring that participants used well-defined counting procedures. The question of interest, then, is whether counting procedures would also be used for the primary time production task. Because of the pacing of the stimuli and the duration of the temporal units, there was no simple mapping of the counts to the number of elapsed units. Specifically, the correspondence between the number of temporal units and the number of stimuli (circles or tones) in the counting task was not a whole number (e.g., in Experiment 1, there were 1.826 temporal units separating each successive secondary-task stimulus). Thus, in light of findings by Killeen and Weiss (1987) that rhythmic counting is the preferred strategy for time judgments, the purpose of using counting as a secondary task was to encourage a counting procedure to be used for time production based on the secondary task but to preclude a simple procedure involving just counting secondary-task stimuli as the main basis for the primary time production task.

To examine transfer of learning, training on the skill of time production with one concurrent secondary task was compared with retraining on that skill with a different secondary task after a 5-min delay. A short delay was used because of the emphasis on transfer, as opposed to retention. Also, because of the focus on transfer rather than retention, only conditions involving changes in the secondary task were used. In the earlier studies by Healy et al. (2005) and Wohldmann et al. (2010), no loss in performance on the primary task was found over a 1-week delay when the secondary-task conditions were not changed and when the intervals were repeated during training and retraining. Thus, it seems safe to assume that performance would show no loss across a 5-min delay under no-change conditions. The eight transfer conditions varied either the modality of the stimuli or the difficulty of the task requirements between training and retraining. On the basis of the context-dependent nature of time production and the attentional models of time production, transfer of training should obtain only when the attentional demands of the concurrent task do not change between training and retraining. However, on the basis of the procedural reinstatement hypothesis, transfer should occur even when the attentional demands are changed, to the extent that the counting procedures used to perform the primary- and secondary-task combination are the same across training and retraining.

\footnotetext{
${ }^{3}$ The two tasks were not described to the participants as "primary" or "secondary," but instructions for the time production task were given first and feedback was provided only for the time production task, not for the counting task.
} 


\section{Method}

\section{Participants}

Eighty University of Colorado undergraduates participated for credit in an introductory psychology course. The participants were assigned by a fixed rotation to one of the eight training and retraining combinations, with 10 participants in each transfer condition.

\section{Design}

Participants were trained and, following a 5-min break, retrained on the time production task for six blocks of six trials, with the same six intervals used in a random order in each block. Four training conditions that differed in the concurrent secondary-task requirements were included. In the easy visual (EV) and easy auditory (EA) conditions, participants were required to count either the total number of circles that appeared on the computer screen or the total number of tones that were played, respectively (i.e., one count was reported at the end of each trial). The difficult visual (DV) and difficult auditory (DA) conditions were identical to the easy conditions, except that participants in those conditions had to count separately the two types of stimuli presented (i.e., two counts were reported at the end of each trial). To examine how difficulty and modality affected learning during training, the design included the factors of training difficulty (easy, difficult) and training modality (visual, auditory). To examine transfer, an additional two-level factor of switch condition was included: switch difficulty, which involved a match in the modality and a switch in the task difficulty between training and retraining, and switch modality, which involved a match in the task difficulty and a switch in the task modality between training and retraining.

Eight transfer conditions changing either between visual and auditory tasks or between easy and difficult tasks were included. A no-change condition was not included because, as was mentioned earlier, in the previous studies by Healy et al. (2005) and Wohldmann et al. (2010), there was no loss in performance between training and retraining even after a 1week delay unless every trial involved a different interval during training. The primary dependent measure used for time production was the same as that used by Healy et al. and Wohldmann et al.- namely, the proportional absolute error of response accuracy for the estimated interval, which is the absolute difference between the estimated interval and the specified interval divided by the specified interval. This measure provides a normalized assessment of error magnitude. Also examined was proportional relative error, which is the signed difference between the estimated interval and the specified interval divided by the specified interval and provides a directional assessment of error bias. Although this measure reveals the participants' bias, it does not illuminate the issues of central concern in the present study, which is focused on specificity and transfer. Thus, a summary of the results for that measure is presented in the Appendix.

In summary, the design for training was a $2 \times 2 \times 6$ mixed factorial including training modality (visual, auditory) and training difficulty (easy, difficult) as between-subjects variables and block (1-6) as a within-subjects variable. The analysis of proportional relative error for time production also included the factor of interval magnitude (short, long). Similar analyses were conducted for retraining, but for simplicity, because they are not germane to the theoretical issues of greatest interest, they are not included here. In addition to the training analyses, a pair of analyses was conducted specifically to examine transfer in terms of proportional absolute error. In a study of speeded aiming, Wohldmann and Healy (2010) found that different measures of transfer yielded different conclusions concerning the presence or absence of transfer, suggesting that, in some cases, there was partial but not full transfer. Separate analyses were conducted to examine any interference or facilitation found from prior training to retraining. A block 6 analysis assessed the extent to which performance at the start of retraining was comparable to that at the end of training, whereas a block 1 analysis assessed the extent to which performance at the start of retraining was comparable to that at the start of training. These analyses included the same factors as those in the training analyses, except that the factor of block was replaced by the factor of phase (training, retraining) and the between-subjects factor of switch condition (switch difficulty, switch modality) was added. All of these analyses used proportional absolute error as the dependent variable.

In addition, an index of transfer was calculated to reflect the degree to which performance improved from training to retraining, with a value equal to (or less than) 0 indicating no transfer, a value equal to (or greater than) 1 indicating full transfer, and a value between 0 and 1 indicating partial transfer. This index is similar to one commonly used in the literature (e.g., Ahissar \& Hochstein, 1997; Jeter, Dosher, Petrov, \& Lu, 2009). The index was calculated as follows:

$$
\begin{aligned}
\text { Transfer }= & {[\text { Block1 }(\text { training })-\text { Block1 }(\text { retraining })] } \\
& /[\text { Block1 }(\text { training })-\text { Block6 }(\text { training })] .
\end{aligned}
$$

This index provides a measure of how much was gained (or lost) from training to retraining as a proportion of how much was gained during training. Because participants were in different modality or difficulty conditions during training and retraining, the training values used to calculate this index for a given participant were mean values based on 
performance during training for all participants in the given combination of training modality and training difficulty. Therefore, for example, for a participant retrained on the DA task, the training values for the transfer index were the average values for all participants trained on the DA task. Thus, the retraining values in this index were individual measures, whereas the training values were group means for the same modality and difficulty conditions as those used in retraining. This index used proportional absolute error as the dependent variable. An analysis of variance (ANOVA) on the transfer index was a $2 \times 2 \times 2$ factorial including the between-subjects factors of training modality, training difficulty, and switch condition.

\section{Materials and apparatus}

Six Macintosh computers were used during both training and retraining. The program was written in REALbasic ${ }^{\circledR}$. The fixed arbitrary intervals of time used during both training and retraining were the same as those used previously (Healy et al., 2005; Wohldmann et al., 2010) (with one unit equal to $783 \mathrm{~ms})$ and were 21 units $(16.443 \mathrm{~s}), 25$ units (19.575 s), 32 units (25.056 s), 47 units (36.801 s), 50 units $(39.150 \mathrm{~s})$, and 56 units $(43.848 \mathrm{~s})$, with the first three less than $30 \mathrm{~s}$ (short) and the last three greater than $30 \mathrm{~s}$ (long).

\section{Procedure}

During training and retraining, participants were given feedback on the actual length of the interval they produced and on the difference between their produced interval and the specified interval, both in the arbitrary units. The instructions for the time production task were presented on the computer screen and were as follows:

You will be asked to estimate a certain interval of time, expressed in fixed arbitrary "units" (not standard units like seconds or minutes). At the beginning of each trial, you will be told the number of units in the interval for you to estimate. Next you will hear a beep. Then when you're ready, press the space bar to indicate that the specified number of units have passed since the beep. . . . You will be given feedback at the end of each trial on the length of the interval in units that you estimated and the difference between that estimated interval and the specified interval. Your task is to try to become as accurate as you can in estimating these time intervals.

Instructions for the secondary tasks were also provided on the computer screen. Participants in the EV condition were told that along with the task of estimating a certain interval of time, they were to count the total number of both large and small circles that appeared on the screen and that, after each trial, they would need to type into one blank space that total number. Participants in the DV condition were asked to count separately each large and each small circle and were told that, after each trial, they would need to type into one blank space the number of large circles and into a second blank space the number of small circles. Participants in the auditory conditions were given similar instructions, except that instead of counting large and small circles, they were told to count low- and high-pitched tones. Note that all participants counted all stimuli but that, in the easy conditions, only a single total count was required, whereas in the difficult conditions, two separate counts, one for each type of stimulus, was required.

The duration of each tone and each circle was $200 \mathrm{~ms}$, and between the offset of one tone or one circle and the onset of the next, there was a 1,230-ms delay. This particular spacing was chosen because it is greater than one unit $(783 \mathrm{~ms})$ but less than two units $(1,566 \mathrm{~ms})$ and so the value of a unit was not completely predictable from the requirements of the secondary task. A random number generator, selecting whole numbers between 1 and 100, determined the size of the circle and the pitch of the tone in the sequence for every trial. If the number generated fell between 1 and 40, a large circle appeared or a low tone was played. If the number fell between 41 and 100, a small circle appeared or a high tone was played. At the start of each trial, the random number generator was given a unique seed (i.e., 36 different seeds) that determined the pattern of the sequence, so that the sequence of high and low tones would be exactly the same for each trial as the sequence of small and large circles (i.e., large circles appeared when low tones played, and small circles appeared when high tones played). This procedure ensured that the sequence of the secondary task would be identical for all participants, regardless of the training modality or level of difficulty. During the 5-min delay between training and retraining, participants sat quietly at their desks.

\section{Results}

\section{Training}

For the repeated measures ANOVA of training data, as well as for all subsequent analyses, all significant $(p<.05)$ main effects and interactions are discussed. The results for proportional absolute error on the time production task are summarized in Fig. 1 as a function of training modality, training difficulty, and block. There was a main effect of training difficulty, $F(1,76)=2.25, M S E=.004, p=.049$, with higher proportional absolute error for participants in the difficult training conditions than for those in the easy training conditions, thereby validating the difficulty manipulation as influencing time production despite the fact that it involved only the counting task. Furthermore, performance on the time production task improved significantly across 


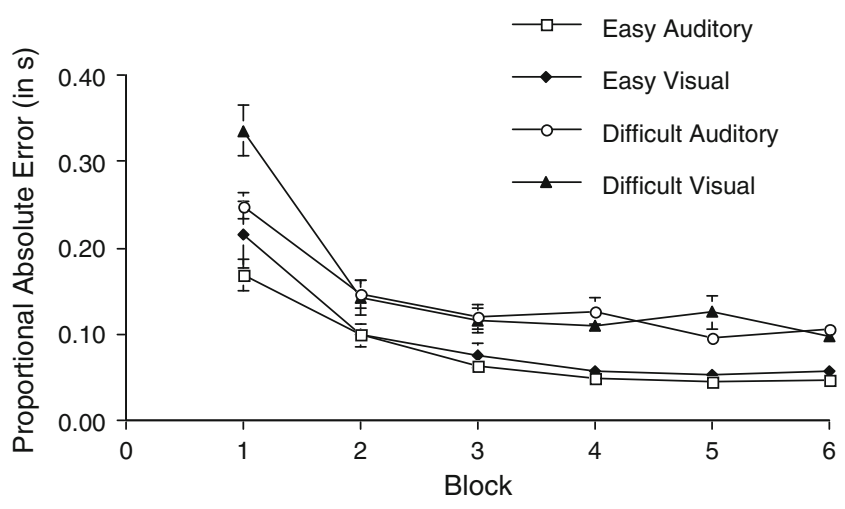

Fig. 1 Mean proportional absolute error on the time production task during training as a function of training modality, training difficulty, and block in Experiment 1

blocks, $F(5,380)=84.91, M S E=.004, p<.001$, especially across the first two blocks of training. Improvement across blocks depended both on training difficulty, $F(5,380)=$ $41.55, M S E=.011, p<.001$, and on training modality, $F$ $(5,380)=3.99, M S E=.004, p=.001$. Specifically, there was greater improvement across blocks for participants who trained with one of the difficult secondary tasks than for those who trained with one of the easy secondary tasks. In addition, there was greater improvement across blocks for participants who trained in the visual modality than for those who trained in the auditory modality.

\section{Transfer}

The transfer analyses involved proportional absolute error and were focused on three blocks (blocks 1 and 6 of training and block 1 of retraining). The results of these analyses are summarized in Fig. 2 in terms of the mean proportional absolute error on those blocks as a function of transfer condition. Block 6 of retraining is also included as a point of comparison, although it did not enter into any of the analyses.

Three analyses were examined, all of which focus on the first block of retraining: block 6 analysis, in which retraining was compared with the last block of training; block 1 analysis, in which retraining was compared, instead, with the first block of training; and the transfer index analysis, which includes both block 1 and block 6 of training. If there is any facilitation from training on initial retraining, the block 1 analysis should reveal less error in retraining than in training, and if there is any interference from training on initial retraining, the block 6 analysis should reveal more error in retraining than in training. The transfer index analysis should reveal whether transfer is partial or complete, relative to the learning that occurred during training.

Block 6 analysis Switching secondary-task requirements between training block 6 and retraining block 1 led to a significant increase in proportional absolute error on the time production task (training $=.077$, retraining $=.093$ ), reflecting interference (negative transfer), $F(1,72)=4.83$, $M S E=.002, p=.031$. Not surprisingly, the main effect of training difficulty was also significant, $F(1,72)=12.53$, $M S E=.004, p<.001$, indicating that, in general, the difficult conditions led to higher proportional absolute error than did the easy conditions. In addition, there was a significant three-way interaction between training difficulty, switch condition, and phase, $F(1,72)=6.24, M S E=.002$, $p=.015$. Specifically, participants who trained with one of the easy secondary tasks and retained the same modality (i.e., switched to a more difficult task) showed a larger increase in proportional absolute error between training and retraining than did all other participants, who showed a much more modest increase (or no increase) in error. The larger increase in this case can be explained by the fact that proportional absolute error is higher in the context of the difficult than of the easy secondary task, presumably because of fewer attentional resources available for the primary, duration estimation task under those circumstances.

Block 1 analysis Unlike the block 6 analysis, switching secondary-task requirements between training and retraining in the block 1 analysis (in which the initial blocks of training
Fig. 2 Mean proportional absolute error for blocks 1 and 6 of training and blocks 1 and 6 of retraining on the time production task as a function of transfer condition in Experiment 1

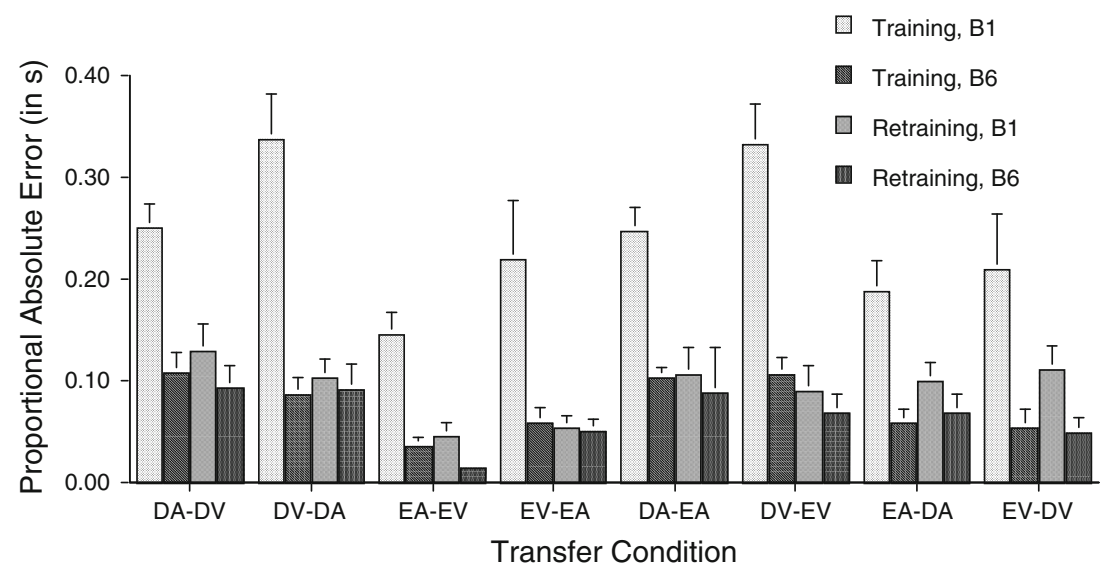


and retraining were compared) led to a large decrease in proportional absolute error on the time production task (training $=.243$, retraining $=.093$ ), suggesting facilitation, $F(1,72)=100.28, M S E=.009, p<.001$. The difficult conditions were, in fact, more difficult (i.e., resulted in higher absolute error) than the easy conditions, $F(1,72)=$ $17.11, M S E=.010, p<.001$. In addition, the interaction between phase and training difficulty, $F(1,72)=5.57, M S E=$ $.009, p=.021$, was significant, demonstrating that difficulty during training affected performance at training itself (difficult $=.293$, easy $=.192$ ) more than performance at retraining (difficult $=.108$, easy $=.078$ ). There was also an interaction between phase and training modality, $F(1,72)=6.07, M S E=.009, p=.016$, reflecting the fact that modality during training affected performance at training itself (auditory $=.209$, visual $=.276$ ) but not performance at retraining (auditory $=.096$, visual $=$ .090). Despite the fact that interference from prior training was evident by the block 6 analysis, a large amount of facilitation from prior training was found in the block 1 analysis.

Transfer index As was mentioned in the Method section, we calculated an index of transfer from the performance levels on the three crucial blocks (blocks 1 and 6 of training and block 1 of retraining), with the index less than (or equal to) 0 when there was no transfer, equal to (or greater than) 1 when there was full transfer, and between 0 and 1 when there was partial transfer. The values of the transfer index for the time production task are summarized in Table 1.

The only significant effect in the analysis of the transfer index was the interaction between switch condition and training difficulty, $F(1,72)=7.44, M S E=.176, p=.008$ (switch modality, difficult training, 0.901; switch modality, easy training, 1.017; switch difficulty, difficult training, 1.007; switch difficulty, easy training, 0.612). This pattern reflects relatively poor transfer when participants switched from an easy task to a difficult task.

Table 1 Mean values of transfer index for time production in Experiments 1 and 2 as a function of transfer condition

\begin{tabular}{lll}
\hline Transfer Condition & Experiment 1 & Experiment 2 \\
\hline DA-DV & 0.829 & 0.931 \\
DV-DA & 0.973 & 0.498 \\
EA-EV & 1.020 & 0.107 \\
EV-EA & 1.015 & 0.271 \\
DA-EA & 0.987 & 1.333 \\
DV-EV & 1.027 & 1.068 \\
EA-DA & 0.570 & 0.693 \\
EV-DV & 0.654 & 0.844 \\
\hline
\end{tabular}

$D A$ difficult auditory; $D V$ difficult visual; $E A$ easy auditory; $E V$ easy visual
Discussion

Experiment 1 was designed to test the hypothesis that transfer of the time production skill can be facilitated by holding constant the pacing of the secondary task both within and across sense modalities. Improvement across blocks of training was evident for all training conditions. Although full positive transfer was not found when the last block of training and the first block of retraining were compared, with significant interference or negative transfer instead, there was large significant partial positive transfer evident in the transfer index and when the first block of training was compared with the first block of retraining. Thus, despite any changes in difficulty (i.e., in attentional demands) that occurred when the secondary-task requirements were altered between training and retraining, participants were able to transfer some of what they had learned during training about the time production task, presumably because of the overlap in the procedures used for time production during training and retraining. This finding is inconsistent with that of Healy et al. (2005), who found no transfer of the time production skill when larger changes to the concurrent secondary task were made between training and retraining, although perfect retention across a 1-week delay was found in that study when the secondary-task conditions were not changed. In the present experiment, the difference between the results for the block 6 analysis, which indicated negative transfer, and the block 1 analysis, which indicated positive transfer, can be understood by the large improvement in performance (decline in proportional absolute error) between the first and the last blocks of training. Although both the block 1 analysis and the block 6 analysis assess transfer by comparing performance at training and retraining, the block 6 analysis uses a much more stringent baseline (the end of training) for assessing transfer than does the block 1 analysis (the start of training).

Two alternative predictions were made at the outset of this experiment. On the basis of the attentional models of time production and because time production is context dependent, the prediction was made that transfer of training would be found only when the attentional demands did not change between training and retraining, which occurred when there was a change in modality. On the other hand, because counting procedures were likely to be used in time production with all of the present secondary tasks, despite the fact that counting stimuli per se could not be directly mapped onto units of time, the prediction was made that transfer of training would be found even when the attentional demands were changed from training to retraining. This second prediction, which was based on the procedural reinstatement principle, was supported by the fact that positive partial transfer was found in all cases by both the analysis of the training index and the block 1 analysis. 


\section{Experiment 2}

In Experiment 1, equal pacing allowed participants to develop an explicit counting strategy that could, presumably, be used for time production even when changes were made to the secondary-task requirements. Experiment 2 examined how minor changes in the counting strategy would affect time production performance by altering the pacing of the secondary tasks across modalities. More specifically, in Experiment 2, tones were presented at a faster rate than circles. Thus, participants who performed either the easy or the difficult tonecounting task were required to count more tones for a given interval of time than were participants who performed either the easy or the difficult circle-counting task. Again, however, within modality, the number of tones and circles was kept constant. The switch in pacing across modalities allowed an assessment of how changes in cognitive demands and, possibly, the counting strategy employed would affect time production performance. Thus, the attentional demands between all four training conditions were different, which offered us the opportunity to test whether changing the attentional demands of the secondary counting task would result in specificity of the time production task or whether, instead, the strategy used for time production is transferable across secondary tasks that differ in attention requirements but that all require counting.

Experiment 2, like Experiment 1, included a $2 \times 2$ design, with separate, crossed manipulations of secondary-task difficulty and secondary-task modality. However, modality was perfectly (and intentionally) confounded with pacing in Experiment 2, and the pacing of the secondary task undoubtedly also influenced task difficulty, because more items would need to be counted when the pacing was more rapid. Thus, Experiment 2, unlike Experiment 1, did not allow for a pure separation of the effects of secondary-task difficulty and modality. However, a comparison of Experiment 1 (where pacing was the same for the two modalities) and Experiment 2 (where pacing was different for the two modalities) should show whether pacing has a significant impact on the extent of transfer.

\section{Method}

\section{Participants}

Eighty undergraduate college students participated for credit in an introductory psychology course. Participants were randomly assigned by a fixed rotation to one of the eight training and retraining combinations, with 10 participants in each of the eight transfer conditions.

\section{Design}

The design for Experiment 2 was the same as that used in Experiment 1. Furthermore, Experiment 2 included the same factors in the analyses, the same dependent measures, and the same transfer index as did Experiment 1.

\section{Materials and apparatus}

The program, computers, and intervals used in Experiment 2 were the same as those used in Experiment 1.

\section{Procedure}

The procedure for Experiment 2 was exactly like that for Experiment 1, except for the pacing of the tones and circles. Specifically, the duration of each circle was $1,000 \mathrm{~ms}$, and the duration of each tone was $200 \mathrm{~ms}$. As in Experiment 1, the delay between the offset of one tone or circle and the onset of the next was $1,230 \mathrm{~ms}$.

Results

\section{Training}

The results for proportional absolute error on time production are summarized in Fig. 3 as a function of training difficulty, training modality, and block. Performance improved significantly across blocks, $F(5,380)=50.25, M S E=.005, p<.001$, especially across the first two blocks of training. In addition, participants in the difficult training conditions had higher proportional absolute error than did those in the easy training conditions, $F(1,76)=21.68, M S E=.018, p<$ .001 . The main effect of training modality was not significant $(F<1)$; however, there was a significant interaction between training difficulty and training modality, $F(1,76)=5.39, M S E=.018, p=.023$. This unexpected effect reflects the fact that with easy tasks, performance in the visual modality was numerically worse (higher absolute error) than in the auditory modality; however, this pattern was reversed with difficult tasks.

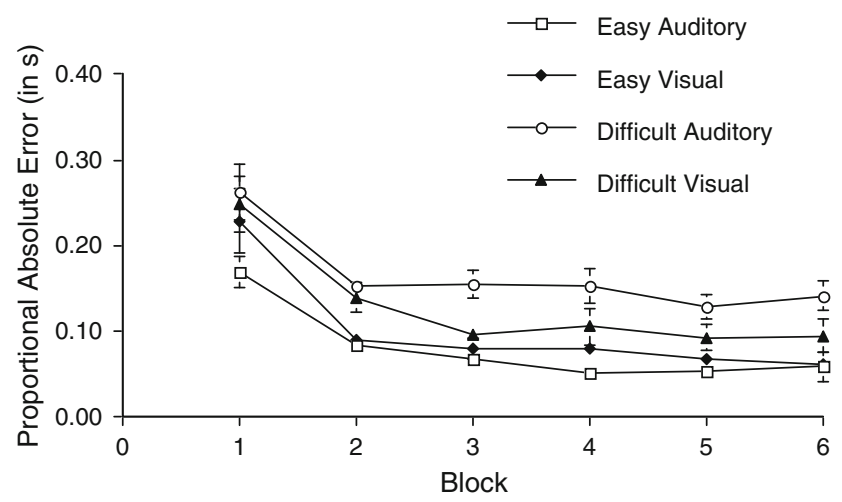

Fig. 3 Mean proportional absolute error on the time production task during training as a function of training modality, training difficulty, and block in Experiment 2 


\section{Transfer}

As in Experiment 1, the transfer analyses involved proportional absolute error on three blocks (blocks 1 and 6 of training and block 1 of retraining). The results of these analyses are summarized in Fig. 4 in terms of the mean proportional absolute error on those blocks as a function of transfer condition. Again, block 6 of retraining is also included as a point of comparison, although it did not enter into any of the analyses.

As for Experiment 1, we calculated an index of transfer from the performance levels on these blocks, with the index equal to (or less than) 0 when there was no transfer, equal to (or greater than) 1 when there was full transfer, and between 0 and 1 when there was partial transfer. The same three sets of analyses of transfer were conducted as in Experiment 1.

Block 6 analysis In general, switching secondary-task requirements between training block 6 and retraining block 1 led to a significant increase in proportional absolute error on the time production task (training $=.089$, retraining $=$ $.129), F(1,72)=14.75, M S E=.004, p<.001$, reflecting interference from training to retraining. That is, practice with one secondary task led to higher errors on the primary task at retraining involving a different secondary task. Participants in the switch modality condition (who changed modalities and, hence, pacing) performed worse than those in the switch difficulty condition, $F(1,72)=9.50, M S E=$ $.011, p=.003$. Furthermore, the interaction of switch condition (switch modality vs. switch difficulty) and phase was significant, $F(1,72)=4.97, M S E=.004, p=.023$; participants in the switch modality condition showed a larger increase in proportional absolute error between training and retraining than did those in the switch difficulty condition. Thus, changing the pacing of the secondary task between training and retraining was important for interference.
Note that, unlike in Experiment 1, there was no significant three-way interaction between training difficulty, switch condition, and phase in Experiment 2. Thus, the important interaction between switch condition and phase is not dependent on training difficulty. Nevertheless, there was a significant interaction between training difficulty and phase, $F(1,72)=8.96, M S E=.004, p=.004$, which reflects a general difficulty of training effect (Schneider, Healy, \& Bourne, 2002). Specifically, training with one of the easy secondary tasks and retraining with any other secondary task led to a larger increase in proportional absolute error across the delay than did training with one of the difficult tasks and retraining with any other task.

A supplemental analysis compared Experiment 1, which involved equal pacing in the visual and auditory modalities, with Experiment 2, which involved unequal pacing in the two modalities. In this analysis, pacing did have an overall impact, reflected in a significant main effect of experiment on proportional absolute error, $F(1,144)=5.87, M S E=$ $.008, p=.017$. In addition, the interaction of experiment and phase approached significance, $F(1,144)=3.44, M S E=$ $.003, p=.066$, because the disadvantage for unequal pacing occurred mainly at the start of retraining (Experiment $1=$ .093 , Experiment $2=.129$ ), rather than at the end of training (Experiment $1=.077$, Experiment $2=.089$ ), suggesting that pacing primarily affects relearning rather than learning.

Block 1 analysis Overall, there was more proportional absolute error during block 1 of training (.228) than during block 1 of retraining (.129); the main effect of phase was significant, $F(1,72)=34.17, M S E=.011, p<.001$, suggesting facilitation from prior training. In addition, the interaction between phase and switch condition was significant, $F$ $(1,72)=7.51, M S E=.011, p=.008$, reflecting the fact that switching modalities between training (.218) and retraining (.166) (which also switched pacing of the secondary task)
Fig. 4 Mean proportional absolute error for blocks 1 and 6 of training and blocks 1 and 6 of retraining on the time production task as a function of transfer condition in Experiment 2

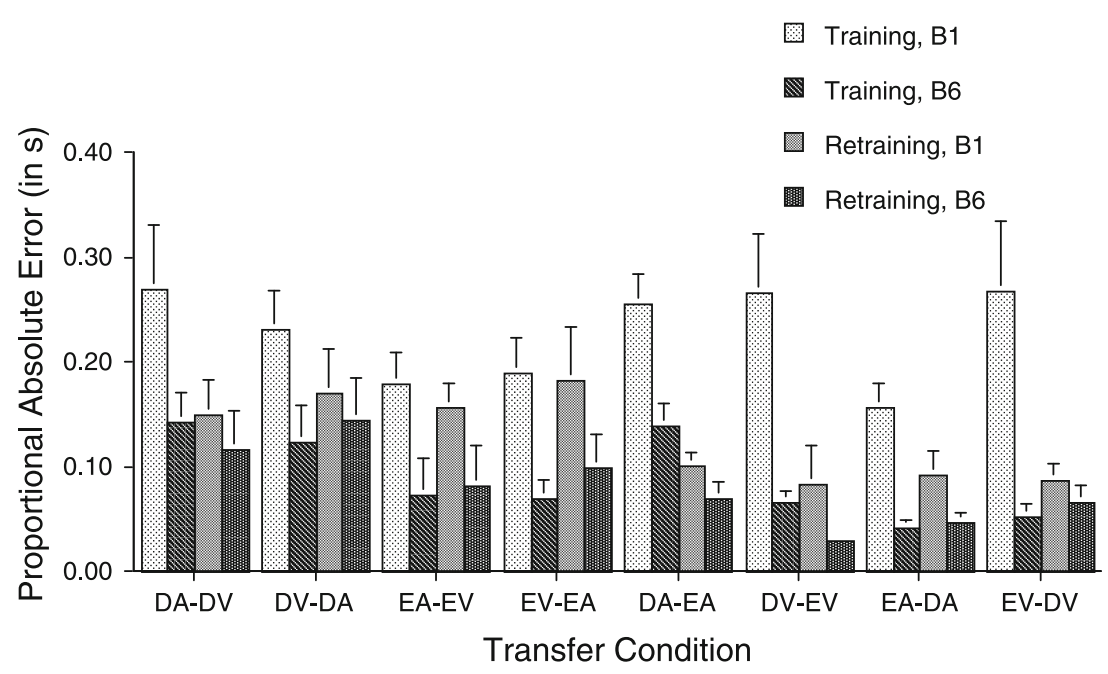


resulted in less improvement than did switching level of difficulty (training $=.237$, retraining $=.092)$ (which maintained the pacing of the secondary task).

As was done for the block 6 analysis, an analysis compared Experiment 1 with Experiment 2. Unlike the results from the block 6 analysis, the main effect of experiment was not significant, $F(1,144)<1$. In addition, the interaction between phase and experiment was, again, only marginally significant, $F(1,144)=3.14, M S E=.010, p=.079$. This finding suggests that the amount of improvement between training block 1 (Experiment $1=.243$, Experiment $2=.196)$ and retraining block 1 (Experiment $1=.118$, Experiment $2=$ .104) tended to be greater with equal pacing (in Experiment 1) than with unequal pacing (in Experiment 2).

Transfer index The values of the transfer index for Experiment 2 are summarized in Table 1. There were two significant effects in the analysis of the transfer index. The main effect of switch condition was significant, $F(1,72)=11.45$, $M S E=0.495, p=.001$, reflecting less transfer when there was a change in modality (i.e., in pacing) (.452) than when there was a change in difficulty (.984). There was also a significant main effect of training difficulty, $F(1,72)=$ 9.27, MSE $=0.495, p=.003$, reflecting more transfer with difficult training (.958) than with easy training (.479). The effect of switch condition underlines the importance of pacing to transfer, and the effect of training difficulty is consonant with the difficulty of training effect (Schneider et al., 2002).

In a supplemental analysis of the transfer index comparing performance in Experiments 1 and 2, there was a significant interaction of experiment and switch condition, $F(1$, $144)=13.86, M S E=.336, p<.001$, reflecting a bigger difference in the amount of transfer between Experiment 1 (with equal pacing of the two modalities) and Experiment 2 (with unequal pacing of the two modalities) when modalities were switched (Experiment $1=.959$, Experiment $2=$ .452 ) than when difficulty was switched (Experiment $1=$ .810 , Experiment $2=.984$ ).

\section{Discussion}

Experiment 2 was designed to test the prediction that holding constant the pacing of the secondary task within sense modalities should facilitate transfer. Unlike in Experiment 1, the pacing was not held constant between modalities. Thus, the counting strategy used to produce intervals when the secondary task involved tone counting was not necessarily appropriate when the secondary task involved circle counting. However, both modalities encouraged a counting strategy for time production that was based on the requirements of a secondary task. In addition, the training conditions differed with respect to the level of difficulty of the concurrent secondary task, which affected primary task performance during training as expected.

Transfer across the delay from the last block of training to the first block of retraining (block 6 analysis) suggested that, at retraining, participants were unable to apply fully the counting strategy acquired during training, showing instead interference (negative transfer) from the prior training. Again, however, significant positive partial transfer of the time production skill was found in the transfer index and when block 1 of training and block 1 of retraining were compared. That is, despite the changes in difficulty that occurred when the secondary-task requirements were altered between training and retraining, participants were able to transfer to retraining some of what had been learned about the time production skill during training. Because less transfer was found when modalities were switched than when levels of difficulty were switched, Experiment 2 provided evidence to suggest that changes in the pacing of the secondary-task stimuli between training and retraining affected temporal productions. This outcome accords well with attentional theories of time production, because a faster pacing should lead to higher cognitive demands of the secondary task, thus depleting resources for the primary temporal production task. Nevertheless, there was substantial positive partial transfer even when the pacing was changed. These results suggest that pacing might be more important to the procedures used for time production than are attentional demands, as would be expected on the basis of the procedural reinstatement principle.

In summary, the present experiment, like Experiment 1, provided a test of two alternative predictions. First, on the basis of attentional theories and the context-dependent nature of time production, the prediction was made that transfer of training would be found in none of the present cases, because, in each instance, the attentional demands changed between training and retraining. Second, on the basis of the overlap in counting procedures used for time production in all the transfer conditions, the prediction was made that some transfer of training would be found in each case and would be greater when pacing was held constant, because of the larger overlap in procedures with constant pacing. The second prediction was clearly supported, thereby providing further evidence for the procedural reinstatement principle on which it was based.

\section{General discussion}

A prospective time production paradigm was used to train and retrain participants on time estimates of durations less than $1 \mathrm{~min}$ in fixed arbitrary units. All participants in this study were trained and retrained while performing a secondary task. Time production is likely to rely on the 
development of some type of counting strategy, perhaps one that is rhythmic, in order to maintain an accurate representation of the number of units and the pacing between those units. Such a counting strategy would require task integration if the secondary task is used as a basis for that strategy in the primary time production task (see Wohldmann et al., 2010). Four training and retraining conditions that differed in either the modality or level of difficulty of the secondary counting task were included in both experiments.

In both experiments, on average, participants significantly improved their time production accuracy across the training blocks. However, during retraining, full positive transfer was generally not evident. Even when the cognitive demands of the primary- and secondary-task combination were held constant across training and retraining, immediate performance of the time production skill usually declined during the 5-min delay, so that error rate was higher on the first block of retraining than on the last block of training. Thus, participants were unable to implement completely the task integration strategy that was developed during training to perform the time production skill in retraining with a different secondary task.

There was, however, large positive partial transfer in both experiments, as reflected both in the transfer index and in the finding of better performance on the first block of retraining than on the first block of training. That is, despite the changes in attentional resources required to perform concurrently the time production and secondary task between training and retraining, participants were able to generalize their counting strategy to a new task that did not necessarily follow the same pacing and imposed different cognitive demands on attention. The pacing of the secondary task had a larger impact on the amount of transfer than did the difficulty of the secondary task in Experiment 2. This finding accords well with the procedural reinstatement principle under the assumption that task integration occurs and that the procedures used for time production rely heavily on the pacing of the secondary-task stimuli (i.e., the counting strategy developed as a basis for time production depends on the rate at which secondary-task stimuli are presented).

If the procedures learned by the participants were unique to each primary- and secondary-task combination, no positive transfer would have been expected. In fact, however, even when the secondary tasks during training and retraining did not match, the strategy acquired during training was partially effective for use in the transfer task. Thus, if the tasks were somehow integrated, the procedures used to perform the functional tasks in training and retraining were not different enough to mask positive partial transfer.

Overall, these findings suggest that time production might not be as sensitive to changes in context as has been indicated in previous research (e.g., Healy et al., 2005).
Furthermore, they support the procedural reinstatement principle of skill learning, which specifies that transfer from one skill to another is dependent on the overlap in procedures between the two skills. In practical terms, finding both task specificity and skill transfer in the present experiments further solidifies the idea presented by Wohldmann and Healy (2010), in a study involving speeded aiming, that these two constructs are not mutually exclusive. Indeed, specificity of learning can be found during retraining even when other findings point to some transfer of what was learned during training. Not only were both specificity and transfer of the time production skill found, but also there was evidence of both negative and positive transfer of the time production skill in each experiment. Thus, when transfer is partial, depending on the measure employed (in this case, on the point during training used to assess the change in performance from training to retraining), training can appear both to facilitate and to interfere with retraining performance.

Author Notes We would like to thank Stacey Wells and Kathleen Shea, our research assistants, for helping to test participants and Niels Taatgen for helpful comments about this research. We are also grateful to two anonymous reviewers, both of whom recommended the use of the index of transfer that we employ here.

This research was supported in part by Army Research Institute Contracts DASW01-99-K-0002 and DASW01-03-K-0002 and by Army Research Office Grant W911NF-05-1-0153 to the University of Colorado.

\section{Appendix Proportional relative error}

For proportion relative error of the primary task, positive bias occurs when the produced interval is longer than the specified interval, and negative bias occurs when the produced interval is shorter than the specified interval. The reported results are limited to training.

\section{Experiment 1}

The results for proportional relative error on the time production task are summarized in Fig. 5. For the measure of proportional relative error, we found a main effect of interval magnitude, $F(1,76)=99.16, M S E=.017, p<.001$, with, on average, positive bias on short intervals (.040) and negative bias on long intervals (-.045). This effect reflects a bias to produce intervals toward the central tendency of the practice range. In addition, this bias on short and long intervals depended on the difficulty of the training task, $F$ $(1,76)=13.47, M S E=.017, p<.001$. Participants in the difficult conditions showed more positive bias on short intervals and more negative bias on long intervals than did participants in the easy conditions (difficult short $=.058$, difficult long $=-.058$, easy short $=.021$, easy long $=-.032$ ). 


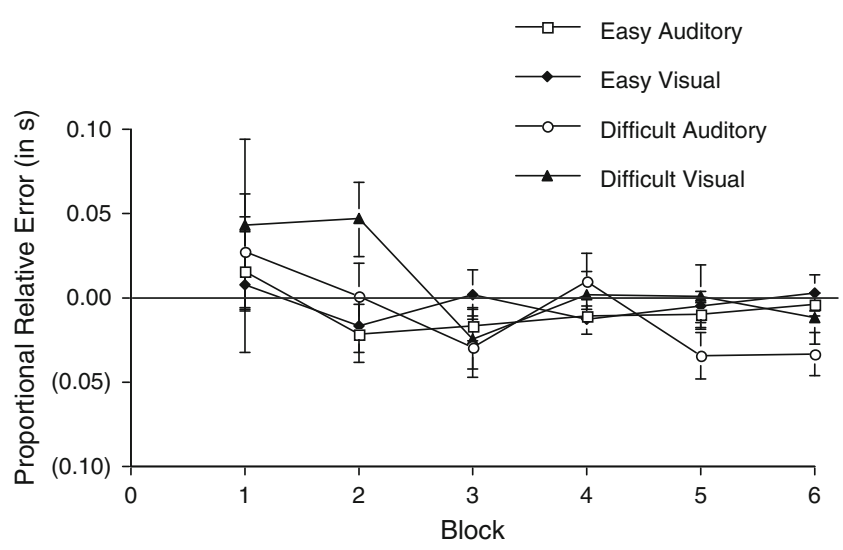

Fig. 5 Mean proportional relative error on the time production task during training as a function of training modality, training difficulty, and block in Experiment 1

Finally, the interaction between interval magnitude and block was significant, $F(5,380)=8.49, M S E=.008, p<$ .001 . Specifically, there was greater improvement across blocks for short intervals (block $1=.100$, block $2=.061$, block $3=.022$, block $4=.027$, block $5=.020$, block $6=.008)$ than for long intervals (block $1=-.053$, block $2=-.055$, block $3=$ -.055 , block $4=-.033$, block $5=-.043$, block $6=-.031$ ).

\section{Experiment 2}

The results for proportional relative error on time production are summarized in Fig. 6. On average, short intervals showed a positive bias $(.028)$, and long intervals showed a negative bias $(-.040), F(1,76)=32.85, M S E=.033, p<.001$. This effect, however, depended on the difficulty of the training task, $F(1,76)=12.69, M S E=.033, p<.001$. Specifically, participants in the difficult conditions showed strong positive bias on short intervals (.056) and strong negative bias on long intervals $(-.053)$, whereas participants in the easy conditions showed no bias on short intervals (-.001) and weak negative bias on long intervals $(-.027)$.

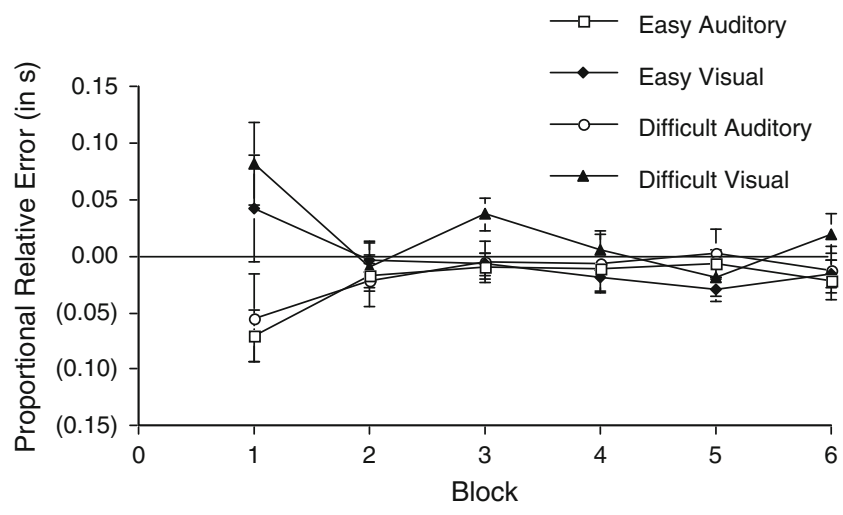

Fig. 6 Mean proportional relative error on the time production task during training as a function of training modality, training difficulty, and block in Experiment 2
Proportional relative error depended on training modality, $F(1,76)=5.75, M S E=.029, p=.019$. Participants in the auditory modality showed negative bias on average (-.019), but those in the visual modality showed positive bias on average (.007). In addition, proportional relative error fluctuated across blocks. Specifically, participants in the auditory conditions showed a strong negative bias at the start of training, with bias becoming less negative across blocks. Participants in the visual conditions showed more variation in bias, with positive bias at the start of training and alternating positive and negative bias thereafter, $F(5,380)=$ $5.59, M S E=.018, p<.001$.

\section{References}

Ahissar, M., \& Hochstein, S. (1997). Task difficulty and the specificity of perceptual learning. Nature, 387, 401-406. doi:10.1038/ $387401 \mathrm{a} 0$

Allan, L. G. (1979). The perception of time. Perception \& Psychophysics, 26, 340-354.

Block, R. A. (1985). Contextual coding in memory: Studies of remembered duration. In J. A. Michon \& J. L. Jackson (Eds.), Time, mind, and behavior (pp. 169-178). Berlin: Springer.

Brown, S. W. (1997). Attentional resources in timing: Interference effects in concurrent temporal and nontemporal working memory tasks. Perception \& Psychophysics, 59, 1118-1140.

Brown, S. W., \& Boltz, M. G. (2002). Attentional processes in time perception: Effects of mental workload and event structure. Journal of Experimental Psychology: Human Perception and Performance, 28, 600-615. doi:10.1037/0096-1523.28.3.600

Clausen, J. (1950). An evaluation of experimental methods of time judgment. Journal of Experimental Psychology, 40, 756-761. doi:10.1037/h0056354

Doane, S. M., Alderton, D. L., Sohn, Y. W., \& Pellegrino, J. W. (1996). Acquisition and transfer of skilled performance: Are visual discrimination skills stimulus specific? Journal of Experimental Psychology: Human Perception and Performance, 22, 12181248. doi:10.1037/0096-1523.22.5.1218

Healy, A. F., \& Bourne, L. E., Jr. (Eds.). (1995). Learning and memory of knowledge and skills: Durability and specificity. Thousand Oaks, CA: Sage.

Healy, A. F., Wohldmann, E. L., Parker, J. T., \& Bourne, L. E., Jr. (2005). Skill training, retention, and transfer: The effects of a concurrent secondary task. Memory \& Cognition, 33, 1457-1471.

Hicks, R. E., Miller, G. W., \& Kinsbourne, M. (1976). Prospective and retrospective judgments of time as a function of amount of information processes. American Journal of Psychology, 89, 719-730. doi: $10.2307 / 1421469$

Hsiao, A. T., \& Reber, A. S. (2001). The dual-task SRT procedure: Fine-tuning the timing. Psychonomic Bulletin \& Review, 8, 336342.

Jeter, P. E., Dosher, B. A., Petrov, A., \& Lu, Z.-L. (2009). Task precision at transfer determines specificity of perceptual learning. Journal of Vision, 9(3, Art. 1), 1-13. doi:10.1167/9.3.1

Killeen, P. R., \& Weiss, N. A. (1987). Optimal timing and the Weber function. Psychological Review, 94, 455-468. doi:10.1037/0033295X.94.4.455

Morris, C. D., Bransford, J. D., \& Franks, J. J. (1977). Levels of processing versus transfer appropriate processing. Journal of Verbal Learning and Verbal Behavior, 16, 519-533. doi:10.1016/ S0022-5371(77)80016-9 
Posner, M. I., \& Boies, S. J. (1971). Components of attention. Psychological Review, 78, 391-408. doi:10.1037/h0031333

Rah, S. K.-Y., Reber, A. S., \& Hsiao, A. T. (2000). Another wrinkle on the dual-task SRT experiment: It's probably not dual task. Psychonomic Bulletin \& Review, 7, 309-313.

Roediger, H. L., III, Weldon, M. S., \& Challis, B. H. (1989). Explaining dissociations between implicit and explicit measures of retention: A processing account. In H. L. Roediger III \& F. I. M. Craik (Eds.), Varieties of memory and consciousness: Essays in honour of Endel Tulving (pp. 3-41). Hillsdale, NJ: Erlbaum.

Rubinstein, J. S., Meyer, D. E., \& Evans, J. E. (2001). Executive control of cognitive processes in task switching. Journal of Experimental Psychology: Human Perception and Performance, 27, 763-797. doi:10.1037/0096-1523.27.4.763

Ruthruff, E., Van Selst, M., Johnston, J. C., \& Remington, R. (2006). How does practice reduce dual-task interference: Integration, automatization, or just stage-shortening? Psychological Research/Psychologische Forschung, 70, 125-142. doi:10.1007/ s00426-004-0192-7

Schmidtke, V., \& Heuer, H. (1997). Task integration as a factor in secondary-task effects on sequence learning. Psychological Research, 60, 53-71. doi:10.1007/BF00419680
Schneider, V. I., Healy, A. F., \& Bourne, L. E., Jr. (2002). What is learned under difficult conditions is hard to forget: Contextual interference effects in foreign vocabulary acquisition, retention, and transfer. Journal of Memory and Language, 46, 419-440. doi:10.1006/jmla.2001.2813

Tulving, E., \& Thomson, D. M. (1973). Encoding specificity and retrieval processes in episodic memory. Psychological Review, 80, 352-373. doi:10.1037/h0020071

Wallace, M., \& Rabin, A. (1960). Temporal experience. Psychological Bulletin, 57, 213-236. doi:10.1037/h0041410

Wohldmann, E. L., \& Healy, A. F. (2010). Exploring specificity of speeded aiming movements: Examining different measures of transfer. Memory \& Cognition, 38, 344-355. doi:10.3758/MC.38.3.344

Wohldmann, E. L., Healy, A. F., \& Bourne, L. E., Jr. (2010). Task integration in time production. Attention, Perception, \& Psychophysics, 72, 1130-1143. doi:10.3758/APP.72.4.1130

Zakay, D., \& Block, R. A. (1997). Temporal cognition. Current Directions in Psychological Science, 6, 12-16. doi:10.1111/1467-8721. ep11512604

Zakay, D., \& Fallach, E. (1984). Immediate and remote time estimation: A comparison. Acta Psychologica, 57, 69-81. doi:10.1016/ 0001-6918(84)90054-4 